University of Wollongong

Research Online

Faculty of Engineering and Information

Faculty of Engineering and Information

Sciences - Papers: Part A

Sciences

$1-1-2015$

A new approach for classification and characterization of voltage dips and swells using 3D polarization ellipse parameters

Mollah R. Alam

University of Wollongong, mra497@uowmail.edu.au

Kashem M. Muttaqi

University of Wollongong, kashem@uow.edu.au

Abdesselam Bouzerdoum

University of Wollongong, bouzer@uow.edu.au

Follow this and additional works at: https://ro.uow.edu.au/eispapers

Part of the Engineering Commons, and the Science and Technology Studies Commons

Research Online is the open access institutional repository for the University of Wollongong. For further information contact the UOW Library: research-pubs@uow.edu.au 


\title{
A new approach for classification and characterization of voltage dips and swells using 3D polarization ellipse parameters
}

\begin{abstract}
This paper presents a new method for classification and characterization of voltage dips and swells in electricity networks. The proposed method exploits unique signatures and parameters of three phase voltage signals extracted from the polarization ellipse in three-dimensional (3D) co-ordinates. Five ellipse parameters, which include azimuthal angle, elevation, tilt, semi-minor axis and semi-major axis, are used to classify and characterize voltage dips and swells. Seven types of voltage dips, which include a total of 19 groups of dips incorporating different kinds of balanced (three-phase dips) and unbalanced (singlephase or double-phase) dips, are identified and successfully classified using the 3D polarization ellipse parameters. Two types of voltage swells, which include a total of 6 groups, are also classified using the proposed method. The proposed method is validated using real measurement data, recorded waveforms provided by the IEEE 1159.2 working group, and the data of unbalanced dips associated with phase angle jumps, voltage drops and rotations due to loading effects.
\end{abstract}

\section{Keywords}

voltage, ellipse, characterization, classification, approach, polarization, 3d, swells, dips, parameters

\section{Disciplines}

Engineering | Science and Technology Studies

\section{Publication Details}

M. R. Alam, K. M. Muttaqi \& A. Bouzerdoum, "A new approach for classification and characterization of voltage dips and swells using 3D polarization ellipse parameters," IEEE Transactions on Power Delivery, vol. 30, (3) pp. 1344-1353, 2015. 


\title{
A New Approach for Classification and Characterization of Voltage Dips and Swells using 3D Polarization Ellipse Parameters
}

\author{
M. R. Alam, Student Member, IEEE, K. M. Muttaqi, Senior Member, IEEE, and A. Bouzerdoum, \\ Senior Member, IEEE
}

\begin{abstract}
This paper presents a new method for classification and characterization of voltage dips and swells in electricity networks. The proposed method exploits unique signatures and parameters of three phase voltage signals extracted from the polarization ellipse in three-dimensional (3D) co-ordinates. Five ellipse parameters, which include azimuthal angle, elevation, tilt, semi-minor axis and semi-major axis, are used to classify and characterize voltage dips and swells. Seven types of voltage dips, which include a total of $\mathbf{1 9}$ groups of dips incorporating different kinds of balanced (three-phase dips) and unbalanced (singlephase or double-phase) dips, are identified and successfully classified using the 3D polarization ellipse parameters. Two types of voltage swells, which include a total of 6 groups, are also classified using the proposed method. The proposed method is validated using real measurement data, recorded waveforms provided by the IEEE 1159.2 working group, and the data of unbalanced dips associated with phase angle jumps, voltage drops and rotations due to loading effects.
\end{abstract}

Index Terms-Classification, characterization, decision boundary, polarization ellipse, voltage dips, and voltage quality.

\section{INTRODUCTION}

$\mathrm{N}$ OW-A-DAYS, power electronic devices exhibiting nonlinear characteristics are used widely in utility grids and on customer premises. This gives rise to several power quality (PQ) issues [1]. One such issue is voltage dip or sag. Voltage dips are the short-duration reduction in rootmean-square (rms) voltage caused by switching and/or starting of electrical motors, generators and bulk loads, transformer energization and faults or short circuits in the power networks [2]. Even though power utilities and customers are exerting extensive efforts to improve the reliability of power networks, it has been very challenging to control the external factors that cause voltage dips. Thus, proper mitigation techniques are desired. However, development of mitigation techniques requires the accurate diagnosis, characterization and classification of voltage dips. Furthermore, classification of voltage dips and swells plays an important role in the assessment of voltage dip ride-through capability and immunity specifications of electrical equipment.

M. R. Alam, K. M. Muttaqi, and A. Bouzerdoum are with the School of Electrical, Computer and Telecommunications Engineering, University of Wollongong, NSW 2522, Australia (Emails: mra497@uowmail.edu.au; kashem@uow.edu.au; bouzer@uow.edu.au).
Voltage dips are usually characterized by the minimum voltage magnitude and total duration [3-6]. According to [7], these standards can be effective for the characterization of single-phase and 3-phase balanced voltage dips; however, these approaches fail to characterize unbalanced voltage dips, including single- and double-phase dips. To resolve this problem, Bollen proposed a seven-type dip classification [8], referred to as $\mathrm{ABC}$ classification. Bollen and Zhang also proposed a symmetrical component based technique to classify and characterize voltage dips [2,9]. In [10], it was reported that the symmetrical component technique has some limitations in characterizing unbalanced voltage sags originating from large dynamic loads. In [11], a space vector method was presented wherein the ellipse inclination angle was used to classify single-phase and double-phase voltage dips. However, this method is not suitable for classification of voltage dips for the case of large phase angle jump.

In this paper, we propose a new technique for classification and characterization of balanced and unbalanced voltage dips, including dips associated with phase angle jump. A multi-stage classification algorithms is developed to identify seven types of voltage dips (A, B, D, F, E, C and G) [8] and two types of voltage swells ( $\mathrm{H}$ and I) [11]. The advantage of this method is that it can cope with large angle jumps, and it can detect both voltage dips and swells.

The paper is organized as follows. Section II describes the behavior of the Polarization Ellipse (PE) parameters under the different types of voltage dips. The proposed approach for the classification and characterization of voltage dips and swells is developed in Section III. Section IV presents the experimental validation and results. Section V concludes the paper.

\section{CHARACTERIZATION OF 3-PHASE VOLTAGE WAVEFORMS USING POLARIZATION ELLIPSE}

In the next subsections, the polarization ellipse is obtained by mapping the three phase voltage signals onto three perpendicular axes of a Cartesian co-ordinate system. Then five ellipse parameters, namely azimuthal angle $(\varphi)$, elevation angle $(\theta)$, tilt angle $(\psi)$, semi-minor axis $\left(A_{y}\right)$ and semi-major axis $\left(A_{x}\right)$ are extracted for different types of voltage dips. Moreover, expressions of $\theta$ and $\varphi$ are developed for seven types of voltage dips. 


\section{A. Polarization Ellipse in 3-D}

The instantaneous three-phase voltage signals received at a monitoring end of a transmission or distribution network can be presented as

$$
v_{\Phi}(t)=\left|V_{\Phi}\right| \cos \left(\omega t+\alpha_{\Phi}\right)
$$

where $\Phi=a, b$, or $c$ denotes phase- $a$, phase- $b$ and phase- $c$, respectively. These instantaneous voltages can be represented by the vectors in 3D space, which are obtained by mapping $v_{a}(t), v_{b}(t)$ and $v_{c}(t)$ on the $\mathrm{X}, \mathrm{Y}$ and $\mathrm{Z}$ co-ordinates, respectively. The tip of the resultant vector, denoted by $\boldsymbol{R}$, traces an ellipse in 3D space, as illustrated in Fig. 1.

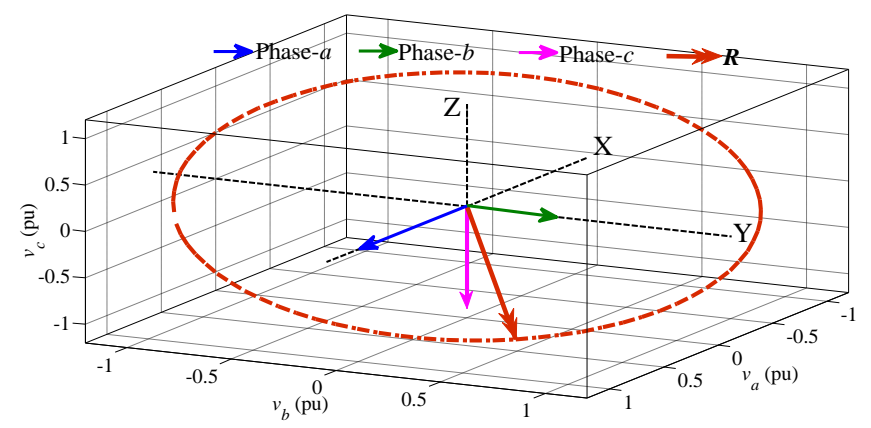

Fig. 1. Instantaneous voltage vectors of phase- $a$, phase- $b$, phase- $c$, and their resultant rotating vector $\boldsymbol{R}$; dash-dot line highlights the locus of the tip of $\boldsymbol{R}$.

The phenomenon of obtaining the plane from the locus of resultant rotating vector $\boldsymbol{R}$ (see Fig. 1) is analogous to the polarization plane obtained from 3D time-varying electromagnetic fields. The parameters of the ellipse can be obtained from the phasor representations of the 3-phase voltages: $\boldsymbol{V}_{a}=\left|\boldsymbol{V}_{a}\right| e^{j \alpha_{a}}, \boldsymbol{V}_{b}=\left|\boldsymbol{V}_{b}\right| e^{j \alpha_{b}}$, and $\boldsymbol{V}_{c}=\left|\boldsymbol{V}_{c}\right| e^{j \alpha_{c}}$. Altogether, these 3 phasors can be presented in a compact form as:

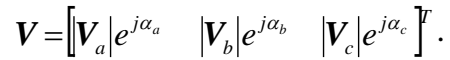

The normal vector $(\boldsymbol{U})$ of the polarization plane is parallel to the cross-product, i.e., $j \boldsymbol{V} \times \boldsymbol{V}^{*}$ [12]. Thus, $\boldsymbol{U}$ is obtained as:

where

$$
\boldsymbol{U} \equiv\left(\boldsymbol{U}_{X}, \boldsymbol{U}_{Y}, \boldsymbol{U}_{Z}\right)
$$

$$
\begin{aligned}
& \boldsymbol{U}_{\boldsymbol{X}}=-2\left|\boldsymbol{V}_{\boldsymbol{b}}\right|\left|\boldsymbol{V}_{\boldsymbol{c}}\right| \sin \left(\alpha_{b}-\alpha_{\boldsymbol{c}}\right) \\
& \boldsymbol{U}_{\boldsymbol{Y}}=-2\left|\boldsymbol{V}_{\boldsymbol{c}}\right|\left|\boldsymbol{V}_{\boldsymbol{a}}\right| \sin \left(\alpha_{\boldsymbol{c}}-\alpha_{\boldsymbol{a}}\right) \\
& \boldsymbol{U}_{\boldsymbol{Z}}=-2\left|\boldsymbol{V}_{\boldsymbol{a}}\right|\left|\boldsymbol{V}_{\boldsymbol{b}}\right| \sin \left(\alpha_{\boldsymbol{a}}-\alpha_{\boldsymbol{b}}\right)
\end{aligned}
$$

From the normal vector $\boldsymbol{U}$, the azimuth angle $\varphi$ and elevation $\theta$ can be derived as (see Fig. 2),

$$
\begin{aligned}
& \theta=\arctan \left(\sqrt{\boldsymbol{U}_{X}^{2}+\boldsymbol{U}_{Y}^{2}} / \boldsymbol{U}_{Z}\right) \\
& \varphi=\arctan \left(\boldsymbol{U}_{Y} / \boldsymbol{U}_{X}\right)
\end{aligned}
$$

Now, using Cartesian to spherical coordinate conversion [13], the transverse signal components are represented as:

$$
\begin{aligned}
& \boldsymbol{S}_{\theta}=\boldsymbol{V}_{\boldsymbol{a}} \cos \theta \cos \varphi+\boldsymbol{V}_{\boldsymbol{b}} \cos \theta \sin \varphi-\boldsymbol{V}_{\boldsymbol{c}} \sin \theta \\
& \boldsymbol{S}_{\varphi}=-\boldsymbol{V}_{\boldsymbol{a}} \sin \varphi+\boldsymbol{V}_{\boldsymbol{b}} \cos \varphi
\end{aligned}
$$

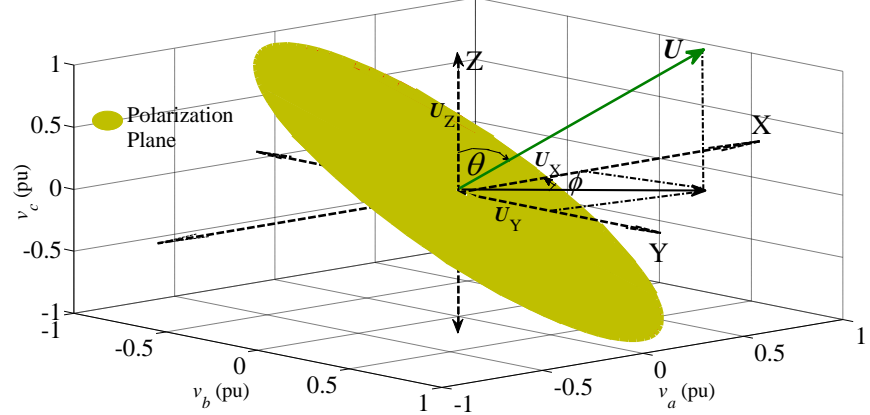

Fig. 2. PE parameters, $\theta$ and $\varphi$, extracted from $3 D$ representation of $3-\Phi$ voltage signals in polarization concept.

With $\boldsymbol{S}_{\theta}$ and $\boldsymbol{S}_{\varphi}$, which are analogous to the two electric field components of a plane electromagnetic wave [14], the elliptical polarization parameters can be obtained from the planar 2D ellipse (see Fig. 3). To extract the ellipse parameters, namely ellipticity, tilt, and the major and minor semi-axes, the Stokes' parameters incorporated in Poincare sphere are used. Equation (6) describes the relationships between the polarization ellipse parameters and Stokes' parameters given in [14]:

$$
\left[\begin{array}{l}
h_{0} \\
h_{1} \\
h_{2} \\
h_{3}
\end{array}\right]=\left[\begin{array}{l}
\left|\boldsymbol{S}_{\theta}\right|^{2}+\left|\boldsymbol{S}_{\varphi}\right|^{2} \\
\left|\boldsymbol{S}_{\theta}\right|^{2}-\left|\boldsymbol{S}_{\varphi}\right|^{2} \\
2\left|\boldsymbol{S}_{\theta}\right| \boldsymbol{S}_{\varphi} \mid \cos \delta \\
2\left|\boldsymbol{S}_{\theta}\right|\left|\boldsymbol{S}_{\varphi}\right| \sin \delta
\end{array}\right]=\left[\begin{array}{l}
h_{0} \\
h_{0} \cos 2 \tau \cos 2 \psi \\
h_{0} \cos 2 \tau \sin 2 \psi \\
h_{0} \sin 2 \tau
\end{array}\right]
$$

where $\delta=\arg \left(\boldsymbol{S}_{\varphi}\right)-\arg \left(\boldsymbol{S}_{\theta}\right), h_{0}, h_{1}, h_{2}, h_{3}$ are the Stokes' parameters, $\tau$ is the ellipticity angle and $\psi$ is the tilt angle. From (6), the tilt angle $(\psi)$ is obtained as:

$$
|\psi|=\left|\frac{1}{2} \arctan \frac{2\left|\boldsymbol{S}_{\theta}\right|\left|\boldsymbol{S}_{\varphi}\right| \cos (\delta)}{\left|\boldsymbol{S}_{\theta}\right|^{2}-\left|\boldsymbol{S}_{\varphi}\right|^{2}}\right|
$$

From Fig. 3 and Eq. (6), the major and minor semi-axes $\left(A_{x}\right)$ and $\left(A_{y}\right)$ can be derived as

$$
\begin{aligned}
& A_{y}=\left|\sqrt{\left|\boldsymbol{S}_{\theta}\right|^{2}+\left|\boldsymbol{S}_{\varphi}\right|^{2}} \sin \left(\frac{1}{2} \arcsin \frac{2\left|\boldsymbol{S}_{\theta}\right|\left|\boldsymbol{S}_{\varphi}\right| \sin (\delta)}{\left|\boldsymbol{S}_{\theta}\right|^{2}+\left|\boldsymbol{S}_{\varphi}\right|^{2}}\right)\right| \\
& A_{x}=\left|\sqrt{\left|\boldsymbol{S}_{\theta}\right|^{2}+\left|\boldsymbol{S}_{\varphi}\right|^{2}} \cos \left(\frac{1}{2} \arcsin \frac{2\left|\boldsymbol{S}_{\theta}\right| \boldsymbol{S}_{\varphi} \mid \sin (\delta)}{\left|\boldsymbol{S}_{\theta}\right|^{2}+\left|\boldsymbol{S}_{\varphi}\right|^{2}}\right)\right|
\end{aligned}
$$

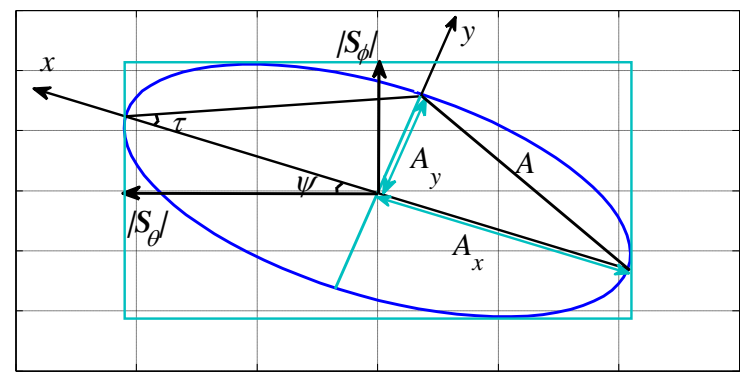

Fig. 3. PE parameters, $A_{y}, A_{x}$ and $\psi$, obtained from classical 2D representation of polarization plane. 
In the next subsection, the five parameters of the 3D PE, namely $\theta, \varphi,|\psi|, A_{y}$ and $A_{x}$, are presented for different dip types.

\section{B. Polarization Ellipse Parameters under seven types of Dips}

In this subsection, the five parameters, $\varphi, \theta,|\psi|, A_{y}$ and $A_{x}$, are used to characterize three-phase voltage dips caused by different types of faults or other incidents (e.g. starting of induction motor). Table I presents the seven types of voltage dips identified in [8]. In this table, the phasor voltages are expressed as a function of the dip-length $d$, and the phasor diagram illustrates the phase voltages before (dotted arrow) and during (solid arrows) the voltage sags or dips.

Due to faults or other disturbances, the phase angle differences among the three phase voltages may deviate from the nominal value of $120^{\circ}$. Moreover, a significant change in phase angle jump could deteriorate the performance of voltage dip classification [10]. Therefore, in the proposed method, the PE parameters are obtained from the projected voltage phasors for a period of one cycle. As an example, the extraction of projected voltage phasors of type $\mathrm{D}$ (see Table I) is demonstrated in Fig. 4. The in-phase and out-of-phase projection of 3 -phase voltages on a-, $\mathrm{b}$ - and c-axis are shown for $d=0.5$. In general, to extract the projected voltages, the three phasors $\left(V_{a} \angle \alpha_{a}, V_{b} \angle \alpha_{b}, V_{c} \angle \alpha_{c}\right)$ are obtained in each cycle, and the angle deviations of $\alpha_{a}, \alpha_{b}$ and $\alpha_{c}$ from a-, b- and c-axis, respectively, are calculated. The in-phase $\left(V_{a_{-} \text {in }}, V_{b_{-} \text {in }}, V_{c_{-} \text {in }}\right)$ and out-of-phase $\left(V_{a_{-} \text {out }}, V_{b_{-} \text {out }}, V_{c_{-} \text {out }}\right)$ projected voltages are obtained as follows:

$$
\begin{aligned}
& \boldsymbol{V}_{a_{-} \text {in }}=\left|\boldsymbol{V}_{a}\right| \cos \left(\alpha_{a}-0\right) \angle 0 \\
& \boldsymbol{V}_{b_{-} \text {in }}=\left|\boldsymbol{V}_{b}\right| \cos \left(\alpha_{b}-4 \pi / 3\right) \angle 4 \pi / 3 \\
& \boldsymbol{V}_{c_{-} \text {in }}=\left|\boldsymbol{V}_{c}\right| \cos \left(\alpha_{c}-2 \pi / 3\right) \angle 2 \pi / 3 \\
& \boldsymbol{V}_{a_{-} \text {out }}=\left|\boldsymbol{V}_{a}\right| \sin \left(\alpha_{a}-0\right) \angle \pi / 2 \\
& \boldsymbol{V}_{b_{-} \text {out }}=\left|\boldsymbol{V}_{b}\right| \sin \left(\alpha_{b}-4 \pi / 3\right) \angle 11 \pi / 6 \\
& \boldsymbol{V}_{c_{-} \text {out }}=\left|\boldsymbol{V}_{c}\right| \sin \left(\alpha_{c}-2 \pi / 3\right) \angle 7 \pi / 6
\end{aligned}
$$

\begin{tabular}{|c|c|c|}
\hline Dip-type & Voltage Phasors & Vector diagram \\
\hline A & $\begin{array}{l}\boldsymbol{V}_{a}=(1-d) \\
\boldsymbol{V}_{b}=-0.5(1-d)(1+j \sqrt{3}) \\
\boldsymbol{V}_{c}=-0.5(1-d)(1-j \sqrt{3})\end{array}$ & \\
\hline B & $\begin{array}{l}\boldsymbol{V}_{a}=(1-d) \\
\boldsymbol{V}_{b}=-0.5(1+j \sqrt{3}) \\
\boldsymbol{V}_{c}=-0.5(1-j \sqrt{3})\end{array}$ & \\
\hline $\mathrm{C}$ & $\begin{array}{l}\boldsymbol{V}_{a}=1 \\
\boldsymbol{V}_{b}=-0.5-0.5(1-d)(j \sqrt{3}) \\
\boldsymbol{V}_{c}=-0.5+0.5(1-d)(j \sqrt{3})\end{array}$ & \\
\hline $\mathrm{D}$ & $\begin{array}{l}\boldsymbol{V}_{a}=(1-d) \\
\boldsymbol{V}_{b}=-0.5(1-d)-0.5(j \sqrt{3}) \\
\boldsymbol{V}_{c}=-0.5(1-d)+0.5(j \sqrt{3})\end{array}$ & \\
\hline E & $\begin{array}{l}\boldsymbol{V}_{a}=1 \\
\boldsymbol{V}_{b}=-0.5(1-d)(1+j \sqrt{3}) \\
\boldsymbol{V}_{c}=-0.5(1-d)(1-j \sqrt{3})\end{array}$ & \\
\hline $\mathrm{F}$ & $\begin{array}{l}\boldsymbol{V}_{a}=(1-d) \\
\boldsymbol{V}_{b}=-0.5(1-d)-\left(\frac{1}{3}+\frac{1-d}{6}\right)(j \sqrt{3}) \\
\boldsymbol{V}_{c}=-0.5(1-d)+\left(\frac{1}{3}+\frac{1-d}{6}\right)(j \sqrt{3})\end{array}$ & $\rightarrow \quad d$ \\
\hline G & $\begin{array}{l}\boldsymbol{V}_{a}=\frac{2}{3}+\frac{1-d}{3} \\
\boldsymbol{V}_{b}=-\frac{1}{3}-\frac{1}{6}(1-d)-0.5(1-d)(j \sqrt{3}) \\
\boldsymbol{V}_{c}=-\frac{1}{3}-\frac{1}{6}(1-d)+0.5(1-d)(j \sqrt{3})\end{array}$ & 1 \\
\hline
\end{tabular}

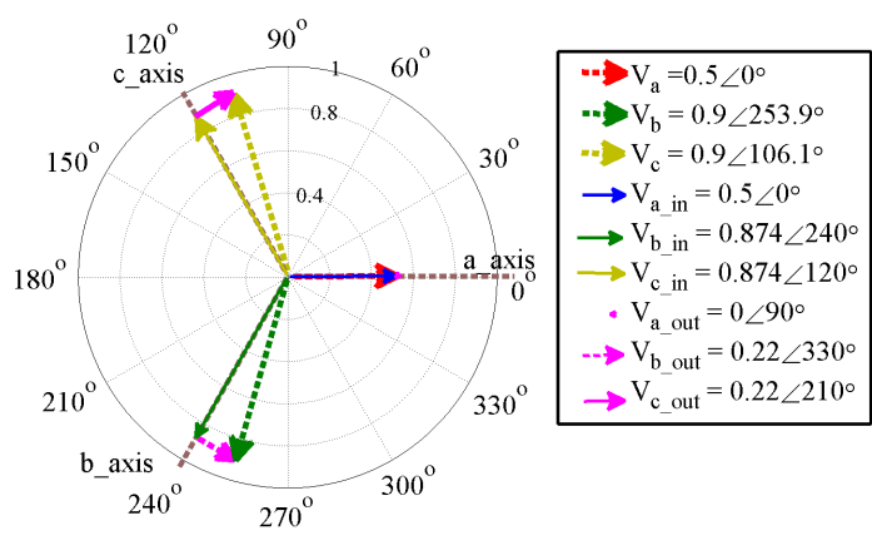

Fig. 4. Extraction of in-phase and out-of-phase projected voltages on a-axis, b-axis and c-axis for dip-type $\mathrm{D}$ with dip-length $d=0.5$.

\begin{tabular}{|c|c|c|c|}
\hline $\begin{array}{l}\text { Affected } \\
\text { dip-phase }\end{array}$ & $\begin{array}{l}\text { Dip } \\
\text { type }\end{array}$ & Elevation angle $(\theta)$ & Azimuthal angle $(\varphi)$ \\
\hline$a b c$ & A & $\pi-\tan ^{-1}(\sqrt{2})$ & $-3 \pi / 4$ \\
\hline$a$ & \multirow{2}{*}{ B } & \multirow{2}{*}{$\pi-\tan ^{-1}\left(\frac{\sqrt{1+(1-d)^{2}}}{1-d}\right.$} & $-\pi+\tan ^{-1}(1-d)$ \\
\hline$b$ & & & $-\pi+\tan ^{-1}(1 /(1-d))$ \\
\hline$a$ & \multirow{2}{*}{$\mathrm{D}$} & \multirow{2}{*}{$\pi-\tan ^{-1}\left(\frac{\sqrt{17 d^{2}-40 d+32}}{4(1-d)}\right)$} & $-\pi+\tan ^{-1}((4-4 d) /(4-d))$ \\
\hline$b$ & & & $-\pi+\tan ^{-1}((4-d) /(4-4 d))$ \\
\hline$a$ & \multirow{2}{*}{$\mathrm{F}$} & \multirow{2}{*}{$\pi-\tan ^{-1}\left(\frac{\sqrt{8-12 d+5 d^{2}}}{2-2 d}\right)$} & $-\pi+\tan ^{-1}((2-2 d) /(2-d))$ \\
\hline$b$ & & & $-\pi+\tan ^{-1}((2-d) /(2-2 d))$ \\
\hline \multirow{3}{*}{$c$} & B & $\pi-\tan ^{-1}(\sqrt{2}-\sqrt{2} d)$ & \multirow{3}{*}{$-3 \pi / 4$} \\
\hline & $\mathrm{D}$ & $\pi-\tan ^{-1}(4 \sqrt{2}(1-d) /(4-d))$ & \\
\hline & $\mathrm{F}$ & $\pi-\tan ^{-1}(2 \sqrt{2}(1-d) /(2-d))$ & \\
\hline \multirow{3}{*}{$a b$} & $\mathrm{E}$ & $\pi-\tan ^{-1}(\sqrt{2} /(1-d))$ & \multirow{3}{*}{$-3 \pi / 4$} \\
\hline & $\mathrm{C}$ & $\pi-\tan ^{-1}(4 \sqrt{2} /(4-3 d))$ & \\
\hline & $\mathrm{G}$ & $\pi-\tan ^{-1}(2 \sqrt{2}(3-d) /(6-5 d))$ & \\
\hline$b c$ & \multirow{2}{*}{$\mathrm{E}$} & \multirow{2}{*}{$\pi-\tan ^{-1}\left(\sqrt{1+(1-d)^{2}}\right)$} & $-\pi+\tan ^{-1}(1 /(1-d))$ \\
\hline$c a$ & & & $-\pi+\tan ^{-1}(1-d)$ \\
\hline$b c$ & \multirow{2}{*}{$\mathrm{C}$} & \multirow{2}{*}{$\pi-\tan ^{-1}\left(\frac{\sqrt{9 d^{2}-24 d+32}}{4}\right)$} & $-\pi+\tan ^{-1}(4 /(4-3 d))$ \\
\hline$c a$ & & & $-\pi+\tan ^{-1}((4-3 d) / 4)$ \\
\hline$b c$ & \multirow{2}{*}{ G } & \multirow{2}{*}{$\pi-\tan ^{-1}\left(\frac{\sqrt{72-84 d+29 d^{2}}}{6-2 d}\right)$} & $-\pi+\tan ^{-1}((6-2 d) /(6-5 d))$ \\
\hline$c a$ & & & $-\pi+\tan ^{-1}((6-5 d) /(6-2 d))$ \\
\hline
\end{tabular}

TABLE I

Phasor Presentation of 3-PHASe Voltages for SEVEn tyPes of Dips

TABLE II

DIP-WISE EXPRESSIONS OF TWO POLARIZATION ELLIPSE PARAMETERS 
The five PE parameters $\left(\theta, \varphi,|\psi|, A_{y}\right.$ and $\left.A_{x}\right)$ are extracted from in-phase projected 3-phase voltages expressed by (10). A sixth PE parameter, denoted as $A_{y}^{\text {out }}$, is extracted from the semi-minor axis obtained from out-of-phase projected 3-phase voltages given in (11). The dip-wise expressions of two PE parameters $(\theta$ and $\varphi)$ are reported in Table II.

In the next section, the algorithm and procedure of classification and characterization of voltage dips and swells are presented.

\section{Classification of Voltage Dips AND Swells}

For the seven types of dips presented in Table I, there are a total of 19 groups (see Table IIIA) covering all possible combinations of dip-affected phase voltages. In Table IIIA, a notation convention has been employed to describe the different voltage dip groups: the letters $a, b$ and $c$ on the left side of the word "dip" indicate the class of dip and the capital letters on the right side indicate the dip-type. For example, $a b \_$dip_E refers to E type dip with severely affected $a b$-phase or class of $a b-\Phi$. Similarly, Table IIIB presents two types of voltage swells, which include 6 swell groups.

TABLE IIIA

GROUPS OF DIPS

\begin{tabular}{|c|c|c|c|c|}
\hline $\begin{array}{c}\text { Serial } \\
\text { No. }\end{array}$ & Groups of dips & $\begin{array}{l}\text { Classes } \\
\text { of dips }\end{array}$ & $\begin{array}{l}\text { Dip- } \\
\text { type }\end{array}$ & $\begin{array}{c}\text { Severely } \\
\text { affected dip- } \Phi\end{array}$ \\
\hline 1 & $a b c \_$dip_A & $\begin{array}{l}a b c-\Phi \\
\text { or 3-Ф }\end{array}$ & A & $a b c$ \\
\hline $2-4$ & $a \_$dip_B,$a \_$dip_D, $a \_$dip_F & $a-\Phi$ & $\mathrm{B}, \mathrm{D}, \mathrm{F}$ & $a$ \\
\hline $5-7$ & $b \_$dip_B, $b$ _dip_D, $b \_$dip_F & $b-\Phi$ & $\mathrm{B}, \mathrm{D}, \mathrm{F}$ & $b$ \\
\hline $8-10$ & $c$ cdip_B, $c$ _dip_D, $c$ _dip_F & $c-\Phi$ & $\mathrm{B}, \mathrm{D}, \mathrm{F}$ & $c$ \\
\hline $11-13$ & $a b \_$dip_E, $a b \_$dip_C, $a b \_$dip_G & $a b-\Phi$ & $\mathrm{E}, \mathrm{C}$ & $a b$ \\
\hline $14-16$ & $b c \_$dip_E, $b c \_$dip_C, $b c \_$dip_G & $b c-\Phi$ & E, C, & $b c$ \\
\hline $17-19$ & ca_dip_E, $c a$ _dip_C, $c a$ dip_G & $c a-\Phi$ & $\mathrm{E}, \mathrm{C}$, & $c a$ \\
\hline
\end{tabular}

TABLE IIIB

GROUPS OF SWELLS

\begin{tabular}{|c|c|c|c|c|}
\hline $\begin{array}{l}\text { Serial } \\
\text { No. }\end{array}$ & Groups of swells & $\begin{array}{l}\text { Group } \\
\text { name }\end{array}$ & $\begin{array}{c}\text { Swell- } \\
\text { type }\end{array}$ & $\begin{array}{l}\text { Severely } \\
\text { affected } \\
\text { swell- } \Phi\end{array}$ \\
\hline 1 & A_swell_I & $a-\Phi$ swell & I & $a$ \\
\hline 2 & $B \_$swell_I & $b$ - $\Phi$ swell & $\mathrm{I}$ & $b$ \\
\hline 3 & C_swell_I & $c$-Ф swell & I & $c$ \\
\hline 4 & $A B \_$swell_H & $a b$ - $\Phi$ swell & $\mathrm{H}$ & $a b$ \\
\hline 5 & $B C \_$swell_H & $b c-\Phi$ swell & $\mathrm{H}$ & $b c$ \\
\hline 6 & CA_swell_H & $c a-\Phi$ swell & $\mathrm{H}$ & $c a$ \\
\hline
\end{tabular}

Numerical values of four PE parameters, $|\psi|, A_{y}, A_{x}$ and $A_{y}^{\text {out }}$, are obtained from Eqs. (2)-(9), by varying the diplength $d$ (from $0.1 \mathrm{pu}$ to $1 \mathrm{pu}$ ). Similarly, the numerical values of the other two PE parameters, $\theta$ and $\varphi$, are acquired from the expressions given in Table II. Thus, six PE parameters are obtained. By analyzing these parameters for $a b c \_$dip_A, it is observed that the 3- $\Phi$ or $a b c$-phase dips can be easily classified by applying a threshold condition on the ratio of the semi-minor to the semi-major axis $\left(A_{y} / A_{x}\right)$ and another threshold condition on semi-minor axis $\left(A_{y}\right)$. According to IEEE 1159.2 standard, a voltage less than $0.9 \mathrm{pu}$ is considered as a dip, whereas a voltage greater than $1.1 \mathrm{pu}$ is defined as swell. In the proposed approach, for a dip-depth equal to $10 \%$ of the nominal voltage i.e. for $d=0.1 \mathrm{pu}$, the ratio of $\left(A_{y} / A_{x}\right)$ is obtained as 0.933 . Taking IEEE 1159.2 standard into account, for minimum allowable voltage magnitude, i.e., for $\left|\boldsymbol{V}_{a}\right|=\left|\boldsymbol{V}_{b}\right|=\left|\boldsymbol{V}_{c}\right|=0.9 \mathrm{pu}, A_{y}$ is obtained as 1.1023 and for maximum allowable voltage limit, i.e., for $\left|\boldsymbol{V}_{a}\right|=\left|\boldsymbol{V}_{b}\right|=\left|\boldsymbol{V}_{c}\right|=1.1$ $\mathrm{pu}, A_{y}$ yields 1.286. Thus, by incorporating the necessary boundary condition on the PE parameters, $A_{x}$ and $A_{y}$, voltage dip, normal condition (no dip) and voltage swell of the system can be classified as shown in Fig. 5. In the next sub-section, an algorithm for classification and characterization of voltage dips is presented. Then, classification of voltage swells is explored in sub-section III-B.

\section{A. Voltage Dip Classification}

The flowchart of Fig. 5 shows the algorithm of dip classification using three phase voltage signals within one cycle window length. As illustrated in Fig. 5, the whole classification methodology is carried out in three stages. In the first stage, $a b c \_$dip_A type is classified by applying the necessary threshold conditions as discussed above. It should be noted that in the first stage, two PE parameters, $A_{y}$ and $A_{x}$ are obtained by considering the actual voltage magnitudes of 3- $\Phi$ voltages; the phase angle differences among the 3-phase voltages are assumed to be separated by $120^{\circ}$.

At the commencement of the second stage, six PE parameters are extracted from in-phase and out-of-phase projected phasors. The dip affected phase is classified in this stage. To this end, $|\psi|$ versus $\varphi$ are considered for six classes of dips: single phase dips $(a-\Phi, b-\Phi$ and $c-\Phi$ dip) and double phase dips ( $a b-\Phi, b c-\Phi$ and $c a-\Phi$ dip). The optimum decision boundaries among these six classes of dips are obtained as the curve bisecting two adjacent regions. To do so, at first, the curves $(|\psi|$ as a function of $\varphi)$ relating the "classes of dips" presented in Table IIIA, are obtained from Eqs. (2)-(5), Eq. (7) and the voltage phasors shown in Table I for $0.1<d<1$. These curves are denoted as $|\psi|_{a}(\varphi),|\psi|_{b}(\varphi),|\psi|_{c}(\varphi)$, $|\psi|_{a b}(\varphi),|\psi|_{b c}(\varphi)$ and $|\psi|_{c a}(\varphi)$, where the different subscripts represent the "classes of dips". As an example, the two curves $|\psi|_{c}(\varphi)$ and $|\psi|_{a b}(\varphi)$ are given by

$$
|\psi|_{c}(\varphi)=\pi / 2 \text {, and }|\psi|_{a b}(\varphi)=0 .
$$

The other four curves $|\psi|_{a}(\varphi),|\psi|_{b}(\varphi),|\psi|_{c a}(\varphi)$ and $|\psi|_{b c}(\varphi)$ are represented by Eq. (7), where the variables $\boldsymbol{S}_{\theta}, \boldsymbol{S}_{\varphi}$ and the range of $\varphi$ are shown in Table IV. Figure 6 illustrates the six decision boundaries separating the different classes. For instance, decision boundary $D_{c-c a}(\varphi)$ separating $c-\Phi$ and $c a-\Phi$ classes is given by

$$
D_{c-c a}(\varphi)=0.5|\psi|_{c}(\varphi)+0.5|\psi|_{c a}(\varphi)
$$

The other five decision boundaries, which include $D_{c-b c}(\varphi), D_{a-a b}(\varphi), D_{b-b c}(\varphi), D_{a-c a}(\varphi)$ and $D_{b-a b}(\varphi)$, are obtained in the same manner. As Fig. 6 shows, if $\varphi<-135^{\circ}$, then $D_{c-c a}(\varphi), D_{a-c a}(\varphi)$ and $D_{a-a b}(\varphi)$ are employed, which implies 
that the class of dip can be $a-\Phi$ or $c-\Phi$ or $c a-\Phi$ or $a b-\Phi$. Similarly, for $\varphi \geq-135^{\circ}$, the decision boundaries $D_{b-b c}(\varphi), D_{b-a b}(\varphi)$, and $D_{c-b c}(\varphi)$ are applied. Thus, six classes of dips are classified.

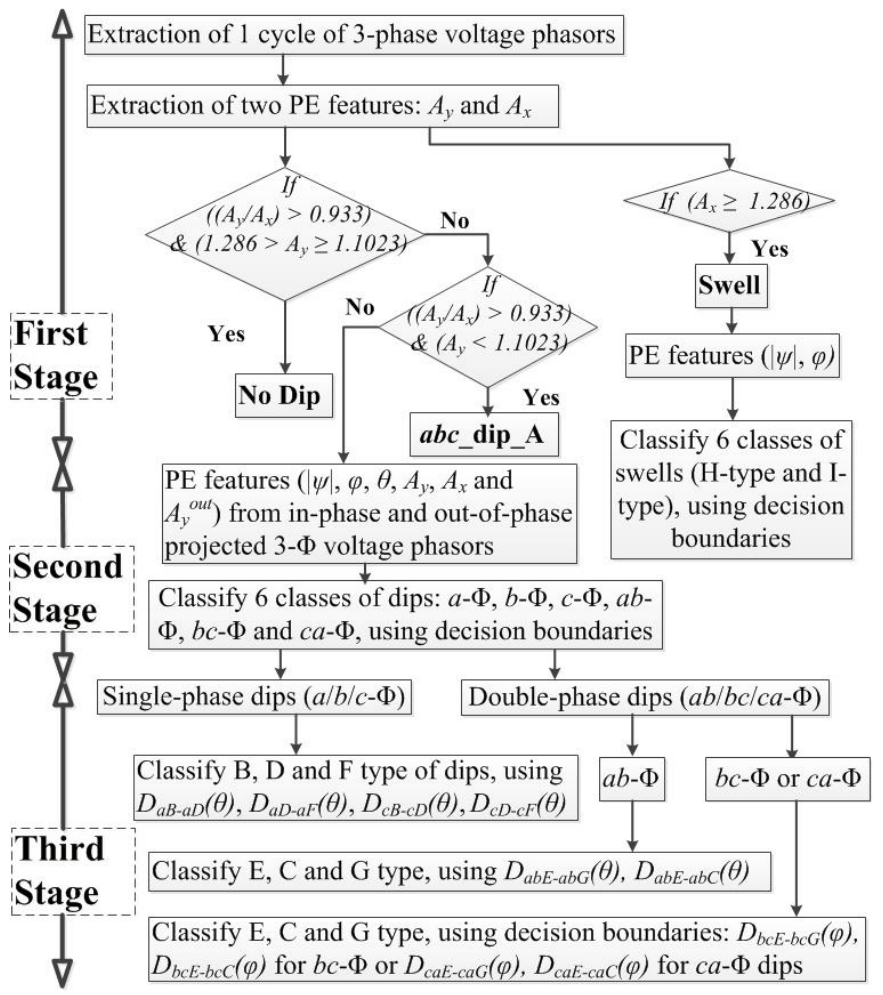

Fig. 5. Flowchart of the proposed method.

TABLE IV

CURVES FOR CLASSIFYING SIX CLASSES OF DIPS

\begin{tabular}{|c|c|c|c|}
\hline Curves & $\boldsymbol{S}_{\theta}$ & $\boldsymbol{S}_{\varphi}$ & $\begin{array}{c}\text { Range } \\
\text { of } \varphi\end{array}$ \\
\cline { 1 - 2 }$|\psi|_{c a}(\varphi)$ & $0.5 \tan \varphi\left\{\sqrt{1+\cos ^{2} \varphi}(1-j \sqrt{3})\right\}$ & $-\frac{0.5\left(1+\sin ^{2} \varphi+j \sqrt{3} \cos ^{2} \varphi\right)}{\cos \varphi}$ & $\begin{array}{l}-180^{\circ} \\
\leq \varphi< \\
-135^{\circ}\end{array}$ \\
\cline { 1 - 2 }$|\psi|_{a}(\varphi)$ & $0.5\left\{\sqrt{1+\sin ^{2} \varphi}(1-j \sqrt{3})\right\}$ & $-\frac{0.5\left(1+\sin ^{2} \varphi+j \sqrt{3} \cos ^{2} \varphi\right)}{\sin \varphi}$ & $\begin{array}{l}-135^{\circ} \\
\leq \varphi \leq \\
-90^{\circ}\end{array}$ \\
\cline { 1 - 2 }$|\psi|_{b}(\varphi)$ & $0.5\left\{\sqrt{1+\cos ^{2} \varphi}(1-j \sqrt{3})\right\}$ & & \\
\cline { 1 - 2 }$\left.|\psi| \psi\right|_{b c}(\varphi)$ & $0.5 \cot \varphi\left\{\sqrt{1+\sin ^{2} \varphi}(1-j \sqrt{3})\right\}$ & &
\end{tabular}

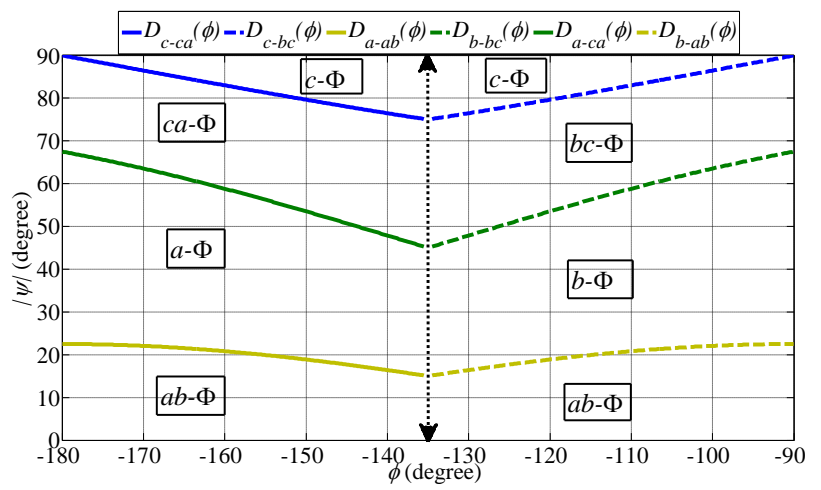

Fig. 6. Decision boundaries, $D_{c-c a}(\varphi), D_{c-b c}(\varphi), D_{a-a b}(\varphi), D_{b-b c}(\varphi), D_{a-c a}(\varphi)$, and $D_{b-a b}(\varphi)$, establishing the classified zone of six classes of dips, which include $a-\Phi, b-\Phi, c-\Phi, a b-\Phi, b c-\Phi$, and $c a-\Phi$; double arrow dotted line acts as a logical separator among the classes of dips.
In the third stage, the dip-type is classified for single- $\Phi$ (B, $\mathrm{D}$ and $\mathrm{F}$ type) and double- $\Phi$ (E, C and $\mathrm{G}$ type) dips. For classifying $\mathrm{B}, \mathrm{D}$ and $\mathrm{F}$ types of single- $\Phi$ dips, the curves $\left(A_{x}\right.$ as a function of $\theta$ ), obtained from Eqs. (2)-(5), Eq. (9) and the phasors of Table I for $0.1<d<1$, are used. The equations of these curves are represented by (9), where the variables $\boldsymbol{S}_{\theta}, \boldsymbol{S}_{\varphi}$ and the range of $\theta$ are reported in Table $\mathrm{V}$. These equations show the behavior of PE parameters $A_{x}$ and $\theta$ under B, D and $\mathrm{F}$ types of single-phase dips. In Table $\mathrm{V}$, the curves, representing $\mathrm{B}, \mathrm{D}$ and $\mathrm{F}$ types of $a-\Phi, b-\Phi$ and $c-\Phi$ dips, are denoted as $A_{x, a B}(\theta), A_{x, a D}(\theta), A_{x, a F}(\theta), A_{x, b B}(\theta), A_{x, b D}(\theta), A_{x, b F}(\theta), A_{x, c B}(\theta)$, $A_{x, c D}(\theta)$ and $A_{x, F}(\theta)$ respectively. Moreover, Fig. 7 shows the decision boundaries, used for the classification of $\mathrm{B}, \mathrm{D}$ and $\mathrm{F}$ types of dips. As an example, the decision boundary $D_{a B-a D}(\theta)$, separating $a \_$dip- $B$ from $a \_$dip_ $D$, is given by

$$
D_{a B-a D}(\theta)=0.5 A_{x, a B}(\theta)+0.5 A_{x, a D}(\theta)
$$

Similarly, the expressions for $D_{a D-a F}(\theta), D_{c B-c D}(\theta)$ and $D_{c D-c F}(\theta)$ are obtained. Using the equations of these decision boundaries, the B, D and F type dips can be easily classified (see Fig. 7).

Double- $\Phi$ dips (E, C and G type) are classified in two steps. First $\mathrm{E}$ and $\mathrm{C}$ type dips are bundled together as $\mathrm{E} / \mathrm{C}$ type, the $\mathrm{E} / \mathrm{C}$ and $\mathrm{G}$ types are classified. In the second step, the dips $\mathrm{E}$ and $\mathrm{C}$ are classified. In order to make a distinction among $\mathrm{E}, \mathrm{C}$ and $\mathrm{G}$ types of $a b-\Phi$ dips, at first, $\mathrm{E} / \mathrm{C}$ and $\mathrm{G}$ type are classified. To do so, $A_{x}$ relating $a b-\Phi$ dips of the E, C, and G types are generated from Eqs. (2)-(5), Eq. (9) and the corresponding phasors of Table I for $0.1<d<1$; these are denoted as $A_{x, a b E}(\theta), A_{x, a b C}(\theta)$ and $A_{x, a b G}(\theta)$ respectively. The curves $A_{x, a b E}(\theta), A_{x, a b c}(\theta)$ and $A_{x, a b G}(\theta)$ are presented by (9), where the variables $\boldsymbol{S}_{\theta}, \boldsymbol{S}_{\varphi}$ and the range of $\theta$ are shown in Table VI. Hence, the decision boundary is expressed as,

$$
D_{a b E-a b G}(\theta)=0.5 A_{x, a b E}(\theta)+0.5 A_{x, a b G}(\theta)
$$

Secondly, the classification of $a b \_$dip_E and $a b \_d i p \_C$ is considered by obtaining the curves derived from Eqs. (2)-(5), Eq. (8) and the corresponding phasors of Table I for $0.1<d<1$. In this case, $A_{y}^{\text {out }}$ is obtained through the proposed $\mathrm{PE}$ technique applied to the out-of-phase phasor voltages. To this end, $A_{y}^{\text {out }}$ related to $a b-\Phi$ dips of type $\mathrm{E}$ and $\mathrm{C}$ type are generated from (8); they are denoted as $A_{y, a b E}^{\text {out }}(\theta)$ and $A_{y, a b C}^{\text {out }}(\theta)$, respectively. Thus, $A_{y, a b E}^{\text {out }}(\theta)$ is obtained as $A_{y, a b E}^{\text {out }}=0$ and $A_{y, a b C}^{\text {out }}(\theta)$ is represented by (8) followed by $\boldsymbol{S}_{\theta}$, $S_{\varphi}$ and the range of $\theta$ as reported in Table VI. The decision boundary is denoted as $D_{a b E-a b C}(\theta)$. The decision boundaries $D_{a b E-a b G}(\theta)$ and $D_{a b E-a b C}(\theta)$, corresponding to the first and second steps of classifying E, C and $\mathrm{G}$ type of $a b-\Phi$ dips, are presented in Figs. 8 (a) and 8 (b, respectively. 
TABLE V

CURVES USED FOR THE CLASSIFICATION OF DIP-TYPE: B, D AND F

\begin{tabular}{|c|c|c|c|}
\hline Curves & $\boldsymbol{S}_{\theta}$ & $S_{\varphi}$ & $\begin{array}{l}\text { Range } \\
\text { of } \theta\end{array}$ \\
\hline$A_{x, a B}(\theta)$ & $0.5(1-j \sqrt{3})$ & $0.5\left(1+j \sqrt{3}\left(1-2 \cos ^{2} \theta\right)\right)$ & \multirow{6}{*}{$\begin{array}{l}90^{\circ} \\
\leq \theta \leq \\
125^{\circ}\end{array}$} \\
\hline$A_{x, b B}(\theta)$ & $\sin \theta$ & $\sin \theta \sqrt{1-2 \cos ^{2} \theta}$ & \\
\hline$A_{x, a D}(\theta)$ & \multirow{2}{*}{$\frac{1.5(1-j \sqrt{3}) \sqrt{1-2 \cos ^{2} \theta}}{\sin \theta\left(4 \sqrt{1-2 \cos ^{2} \theta}+\cos \theta\right)}$} & \multirow{2}{*}{$\frac{1.5(1+j \sqrt{3})\left(1-2 \cos ^{2} \theta\right)}{\sin \theta\left(4 \sqrt{1-2 \cos ^{2} \theta}+\cos \theta\right)}$} & \\
\hline$A_{x, b D}(\theta)$ & & & \\
\hline$A_{x, a F}(\theta)$ & $1.5(1-j \sqrt{3}) \sqrt{1-2 \cos ^{2} \theta}$ & $1.5(1+j \sqrt{3})\left(1-2 \cos ^{2} \theta\right)$ & \\
\hline$A_{x, b F}(\theta)$ & $\sin \theta\left(2 \sqrt{1-2 \cos ^{2} \theta}+\cos \theta\right)$ & $\sin \theta\left(2 \sqrt{1-2 \cos ^{2} \theta}+\cos \theta\right)$ & \\
\hline$A_{x, c B}(\theta)$ & $-0.5(1-j \sqrt{3}) /(\sqrt{2} \cos \theta)$ & $0.5 \times \sqrt{1.5} \times(\sqrt{3}+j)$ & \multirow{5}{*}{$\begin{array}{l}125^{\circ} \\
<\theta \leq \\
180^{\circ}\end{array}$} \\
\hline \multirow{2}{*}{$A_{x, c D}(\theta)$} & $1.5(1-j \sqrt{3})$ & \multirow{2}{*}{$\frac{1.5 \cos \theta(3+j \sqrt{3})}{4 \sqrt{2} \cos \theta+\sin \theta}$} & \\
\hline & $-\overline{4 \sqrt{2} \cos \theta+\sin \theta}$ & & \\
\hline \multirow{2}{*}{$A_{x, c F}(\theta)$} & $-0.5(1-j \sqrt{3})$ & \multirow{2}{*}{$\frac{0.5 \cos \theta(3+j \sqrt{3})}{2 \sqrt{2} \cos \theta+\sin \theta}$} & \\
\hline & $\overline{2 \sqrt{2} \cos \theta+\sin \theta}$ & & \\
\hline
\end{tabular}

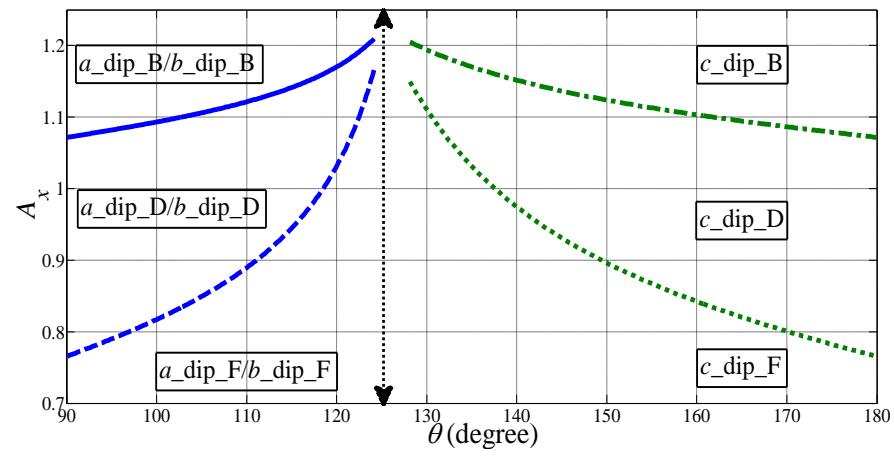

Fig. 7. Decision boundaries, $D_{a B-a D}(\theta)$ (solid line), $D_{a D-a F}(\theta)$ (dash line), $D_{c B-c D}(\theta)$ (dash dotted line), $D_{c D-c F}(\theta)$ (dotted line) establishing the classified zone of B, D and F types of single- $\Phi$ dips; double arrow dotted line acts as a logical separator between $a / b$ - $\Phi$ class and $c$ - $\Phi$ class of dips.

TABLE VI

CURVES USED FOR CLASSIFYING E, C AND G TYPE OF AB- $\Phi$ DIPS

\begin{tabular}{|c|c|c|c|}
\hline Curves & $\boldsymbol{S}_{\theta}$ & $S_{\varphi}$ & $\begin{array}{l}\text { Range } \\
\text { of } \theta\end{array}$ \\
\hline$A_{x, a b E}(\theta)$ & \multirow{2}{*}{$0.5(1-j \sqrt{3}) / \sin \theta$} & \multirow{2}{*}{$-0.5 \times \cos \theta(3+j \sqrt{3}) / \sin \theta$} & \multirow{6}{*}{$\begin{array}{l}90^{\circ} \\
\leq \theta \leq \\
125^{\circ}\end{array}$} \\
\hline$A_{x, a b C}(\theta)$ & & & \\
\hline \multirow{2}{*}{$A_{x, a b G}(\theta)$} & $1.5(1-j \sqrt{3})$ & \multirow{2}{*}{$-\frac{1.5 \cos \theta(3+j \sqrt{3})}{2 \sqrt{2} \cos \theta+5 \sin \theta}$} & \\
\hline & $2 \sqrt{2} \cos \theta+5 \sin \theta$ & & \\
\hline \multirow[t]{2}{*}{$A_{y, a b C}^{\text {out }}(\theta)$} & $\sin \theta+\sqrt{2} \cos \theta$ & $0.5(1+j \sqrt{3})(\sin \theta+\sqrt{2} \cos \theta)$ & \\
\hline & $\sqrt{3} \sin \theta$ & $\sqrt{3} \sin \theta$ & \\
\hline
\end{tabular}

(a)

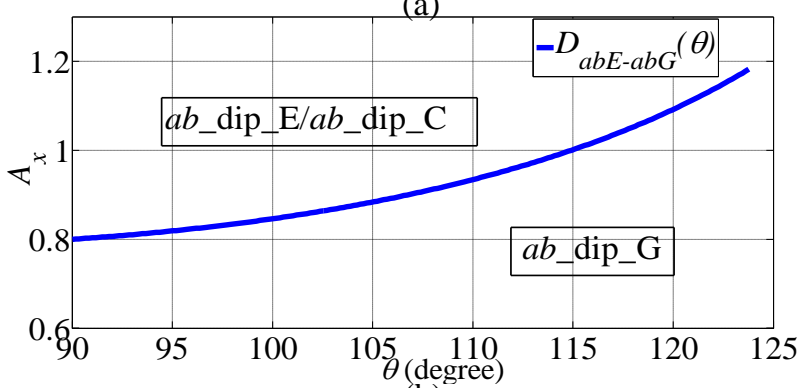

(b)

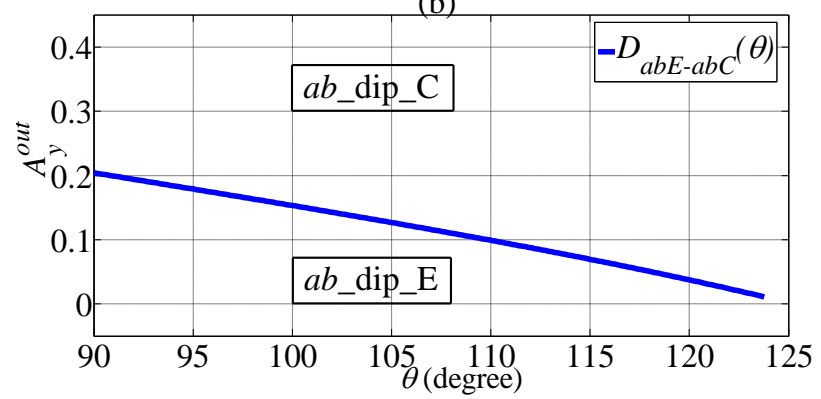

Fig. 8. Classified zone of $a b-\Phi$ dips using decision boundaries: (a) $D_{a b E-a b G}(\theta)$ for $\mathrm{E} / \mathrm{C}$ and $\mathrm{G}$ types; (b) $D_{a b E-a b C}(\theta)$ for $\mathrm{E}$ and $\mathrm{C}$ types.

Following similar steps for classifying $a b-\Phi$ dips of type E, $\mathrm{C}$ and $\mathrm{G}$, the $b c-\Phi$ and $c a-\Phi$ dips are distinguished by the decision boundaries obtained from the curves: $A_{x, b c E}(\varphi), A_{x, b c C}(\varphi), A_{x, b c G}(\varphi), A_{x, c a E}(\varphi), A_{x, c a C}(\varphi), A_{x, c a G}(\varphi)$, $A_{y, b c E}^{\text {out }}(\varphi), A_{y, b c C}^{\text {out }}(\varphi), A_{y, c a E}^{\text {out }}(\varphi)$ and $A_{y, c a C}^{\text {out }}(\varphi)$. It should be noted that $A_{y, b c E}^{\text {out }}(\varphi)$ and $A_{y, c a E}^{\text {out }}(\varphi)$ are obtained as $A_{y, b c E}^{\text {out }}=A_{y}^{\text {out }}, a=0$. The rest of the curves are derived from Eqs. (8) and (9), and the variables presented in Table VII.

TABLE VII

CURVES USED FOR CLASSIFYING E, C AND G TYPE OF BC- $\Phi$ AND CA- $\Phi$ DIPS

\begin{tabular}{|c|c|c|c|}
\hline Curves & $S_{\theta}$ & $S_{\varphi}$ & $\begin{array}{c}\text { Range } \\
\text { of } \varphi\end{array}$ \\
\hline$A_{x, b c E}(\varphi)$ & \multirow{2}{*}{$\frac{(1-j \sqrt{3}) \cos \varphi \sqrt{1+\sin ^{2} \varphi}}{2 \sin \varphi}$} & \multirow{2}{*}{$-\frac{1+j \sqrt{3} \cos ^{2} \varphi+\sin ^{2} \varphi}{2 \sin \varphi}$} & \multirow{4}{*}{$\begin{array}{l}-\frac{3 \pi}{4} \\
<\varphi \leq \\
-\frac{\pi}{2}\end{array}$} \\
\hline$A_{x, b c c}(\varphi)$ & & & \\
\hline$A_{x, b c G}(\varphi)$ & $-\frac{1.5(1-j \sqrt{3}) \cos \varphi \sqrt{1+\sin ^{2} \varphi}}{2 \cos \varphi-5 \sin \varphi}$ & $-\frac{1.5 \cos \theta(3+j \sqrt{3})}{2 \sqrt{2} \cos \theta+5 \sin \theta}$ & \\
\hline$A_{y, b c c}^{o u t}(\varphi)$ & $\frac{\sqrt{3}(1-j \sqrt{3})(\sin \varphi-\cos \varphi)}{6 \sin \varphi}$ & $\frac{\sqrt{3}(1+j \sqrt{3})(\sin \varphi-\cos \varphi)}{6 \sin \varphi}$ & \\
\hline$A_{x, c a E}(\varphi)$ & \multirow{2}{*}{$0.5(1-j \sqrt{3}) \tan \varphi \sqrt{1+\cos ^{2} \varphi}$} & \multirow{2}{*}{$-\frac{\left(1+j \sqrt{3} \cos ^{2} \varphi+\sin ^{2} \varphi\right)}{2 \cos \varphi}$} & \multirow{4}{*}{$\begin{array}{l}-\pi \\
\leq \varphi \leq \\
-\frac{3 \pi}{4}\end{array}$} \\
\hline$A_{x, c a C}(\varphi)$ & & & \\
\hline$A_{x, c a G}(\varphi)$ & $\frac{1.5(1-j \sqrt{3}) \sin \varphi \sqrt{1+\cos ^{2} \varphi}}{5 \cos \varphi-2 \sin \varphi}$ & $-\frac{1.5\left(1+j \sqrt{3} \cos ^{2} \varphi+\sin ^{2} \varphi\right)}{5 \cos \varphi-2 \sin \varphi}$ & \\
\hline$A_{y, \text { ouc }}^{\text {out }}(\varphi)$ & $\frac{\sqrt{3}(1-j \sqrt{3})(\cos \varphi-\sin \varphi)}{6 \cos \varphi}$ & $\sqrt{3}(\cos \varphi-\sin \varphi) / 3 \cos \varphi$ & \\
\hline
\end{tabular}




\section{B. Voltage Swell Classification}

The proposed method is used for classification of voltage swells, which include two types of voltage swells: H- and Itype, as reported in [11] and presented in Table VIII. The Itype swell includes the single- $\Phi$ voltage swells, $a-\Phi, b-\Phi$, and $c-\Phi$ swells, whereas the H-type includes the double- $\Phi$ voltage swell, i.e., $a b-\Phi, b c-\Phi$, and $c a-\Phi$ swell, as shown in Table IIIB. As shown in Fig. 5, the six swell groups are classified by following similar approach to that of classifying six classes of voltage dips. To this end, the equations required for the decision boundaries are denoted as $|\psi|_{A}(\varphi),|\psi|_{B}(\varphi)$, $|\psi|_{C}(\varphi),|\psi|_{A B}(\varphi),|\psi|_{B C}(\varphi)$ and $|\psi|_{C A}(\varphi)$, where subscript letter " $A$ " represents $a-\Phi$ swell, “ $B$ ” represents $b-\Phi$ swell and so on. The equations $(|\psi|$ as a function of $\varphi)$ relating the "groups of swells" presented in Table IIIB, are obtained from Eqs. (2)(5), Eq. (7) and the voltage phasors shown in Table VIII. Thus, the Eqs. of two curves $|\psi|_{A B}(\varphi)$ and $|\psi|_{C}(\varphi)$ are derived as: $|\psi|_{A B}(\varphi)=\pi / 2$, and $|\psi|_{C}(\varphi)=0$. The other four curves, $|\psi|_{A}(\varphi),|\psi|_{B}(\varphi),|\psi|_{C A}(\varphi)$ and $|\psi|_{B C}(\varphi)$ are represented by (7), where the variables $\boldsymbol{S}_{\theta}, \boldsymbol{S}_{\varphi}$ and the range of $\varphi$ are shown in Table IX. From these expressions, the equations of decision boundaries, which bisect two adjacent swell regions, are obtained; see Fig. 9 for illustration. The six groups of swells are classified using the expressions of these decision boundaries.

TABLE VIII

Phasor Presentation of 3-Phase Voltages for Two types of Swells

\begin{tabular}{|c|c|}
\hline Swell-type & Voltage Phasors \\
\hline \multirow{3}{*}{$\mathrm{H}$} & $\boldsymbol{V}_{\boldsymbol{a}}=(1-d)$ \\
& $\boldsymbol{V}_{\boldsymbol{b}}=-0.5(1+d)-0.5(j \sqrt{3})$ \\
& $\boldsymbol{V}_{\boldsymbol{c}}=-0.5(1+d)+0.5(j \sqrt{3})$ \\
\hline \multirow{3}{*}{$\mathrm{I}$} & $\boldsymbol{V}_{\boldsymbol{a}}=(1+2 d)$ \\
& $\boldsymbol{V}_{\boldsymbol{b}}=-0.5(1-d)-0.5(j \sqrt{3})$ \\
& $\boldsymbol{V}_{\boldsymbol{c}}=-0.5(1-d)+0.5(j \sqrt{3})$ \\
\hline
\end{tabular}

TABLE IX

CURVES FOR CLASSIFYING SIX GROUPS OF SWELLS

\begin{tabular}{|c|c|c|c|}
\hline Curves & $\boldsymbol{S}_{\theta}$ & $\boldsymbol{S}_{\varphi}$ & $\begin{array}{c}\text { Range } \\
\text { of } \varphi\end{array}$ \\
\hline$|\psi|_{B}(\varphi)$ & $\frac{1.5 \sin \varphi\left\{\sqrt{1+\cos ^{2} \varphi}(1-j \sqrt{3})\right\}}{\cos \varphi+2 \sin \varphi}$ & $-\frac{1.5\left(1+\sin ^{2} \varphi+j \sqrt{3} \cos ^{2} \varphi\right)}{\cos \varphi+2 \sin \varphi}$ & \multirow{2}{*}{$\begin{array}{l}-180^{\circ} \\
\leq \varphi< \\
-135^{\circ}\end{array}$} \\
\hline$|\psi|_{B C}(\varphi)$ & $0.5\left\{\sqrt{1+\sin ^{2} \varphi}(1-j \sqrt{3})\right\}$ & $-0.5\left(1+\sin ^{2} \varphi+j \sqrt{3} \cos ^{2} \varphi\right) / \cos \varphi$ & \\
\hline$|\psi|_{C A}(\varphi)$ & $0.5\left\{\sqrt{1+\cos ^{2} \varphi}(1-j \sqrt{3})\right\}$ & $-0.5\left(1+\sin ^{2} \varphi+j \sqrt{3} \cos ^{2} \varphi\right) / \sin \varphi$ & \multirow{2}{*}{$\begin{array}{l}-135^{\circ} \\
\leq \varphi \leq \\
-90^{\circ}\end{array}$} \\
\hline$|\psi|_{A}(\varphi)$ & $\frac{1.5 \cos \varphi\left\{\sqrt{1+\sin ^{2} \varphi}(1-j \sqrt{3})\right\}}{2 \cos \varphi+\sin \varphi}$ & $-\frac{1.5\left(1+\sin ^{2} \varphi+j \sqrt{3} \cos ^{2} \varphi\right)}{2 \cos \varphi+\sin \varphi}$ & \\
\hline
\end{tabular}

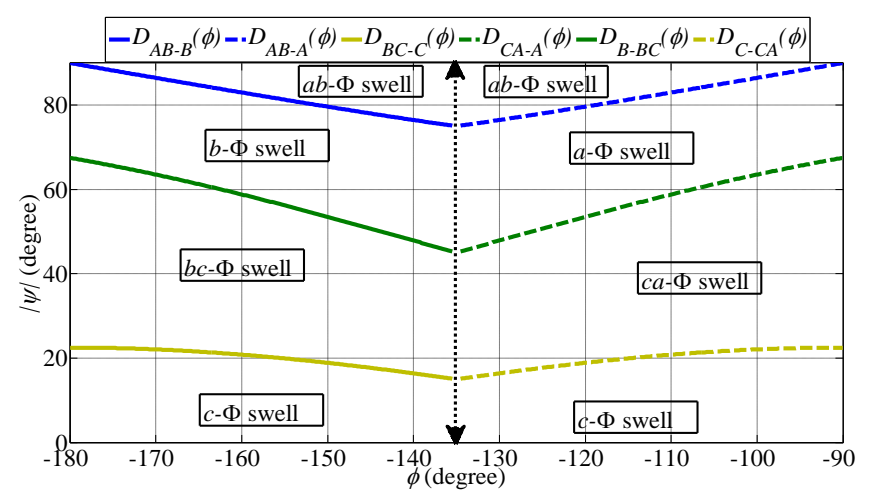

Fig. 9. Decision boundaries, $D_{A B-B}(\varphi), D_{A B-A}(\varphi), D_{B C-C}(\varphi), D_{C A-A}(\varphi), D_{B-B C}(\varphi)$, and $D_{C-C A}(\varphi)$, establishing the classified zone of six groups of swells, which include $a-\Phi, b-\Phi, c-\Phi, a b-\Phi, b c-\Phi$, and $c a-\Phi$; double arrow dotted line acts as a logical separator among the groups of swells.

\section{VALIDATION AND TeSt Results}

The proposed method is validated with recorded waveforms provided by IEEE 1159.2 [15], real measurement data given in [16], and unbalanced dips associated with phase angle jump.

\section{A. Dip classification from Recorded Waveforms}

IEEE 1159.2 working group recorded several test waveforms, which include balanced and unbalanced voltage sags influenced by industrial power electronic equipment [15]. Most of these recorded waveforms are used to validate the proposed method.

The phase voltages may be influenced by noise and harmonic distortion due to the presence of power electronic devices and other electrical equipment. The impact of noise can be seen in Fig. 10, which shows one of the recorded waveforms wave 15 [15]. To extract the ellipse parameters, the DFT (Discrete Fourier Transform) is applied to one cycle long window. The phase voltage magnitude and phase angle at the fundamental frequency (in this case $\pm 60 \mathrm{~Hz}$ ), are extracted and passed through the proposed polarization ellipse technique for dip classification. The proposed algorithm operates on a sliding time-window of 1 cycle length and each of the recorded waveforms has 3-phase voltage signals of six cycle duration. Therefore, if voltage dip or swell is found within any window frame passing through the six cycles of voltage signal, it is detected; otherwise it is classified as normal condition. However, to test the proposed method, normal condition of the recorded waveforms is not shown; only the classification results within one cycle window frame, starting at the inception of voltage sags, are considered and presented in Table X. For instance, 3- $\Phi$ voltage phasors of third cycle ( 0.033 to $0.05 \mathrm{sec}$ duration) are considered for the voltage dipclassification of wave 15 and marked as classified window. Similarly, voltage phasors of other recorded waveforms are classified as shown in Table $\mathrm{X}$.

It is observed that the test recorded waveforms are suffered from voltage dips in single-phase or two-phases or 3-phases; the ground-truth for the "classes of dips" is presented in column 2 of Table X. These recorded waveforms are passed through the proposed multi-stage classification algorithm. Firstly, the PE parameters are extracted for each of the 
recorded waveforms, see columns 3-6 of Table X. Then, using the decision boundaries as highlighted in Fig. 6 and the PE parameters $|\psi|$ and $\varphi$ of Table $\mathrm{X}$, classes of dips are identified. For wave 12, abc- $\Phi$ or 3- $\Phi$ balanced dip is identified by applying the conditions on PE parameters $\left(A_{y} / A_{x}\right)$ and $A_{y}$, see Fig. 5. The classification results, as presented in $7^{\text {th }}$ column of Table X, specify the successful classification of all the test recorded waveforms with $100 \%$ accuracy. In summary, the proposed algorithm is able to provide the exact "classes of dips" as reported in the results of recorded waveforms.

TABLE $X$

CLASSIFICATION OF VOLTAGE DIPS WITH RECORDED WAVEFORMS

\begin{tabular}{|c|c|c|c|c|c|c|}
\hline \multirow{2}{*}{$\begin{array}{c}\text { Wave } \\
\text { number }\end{array}$} & $\begin{array}{c}\text { Classes of } \\
\text { dips } \\
\text { (ground- } \\
\text { truth) }\end{array}$ & \multicolumn{2}{|c|}{ Polarization Ellipse parameters } & $\begin{array}{c}\text { Test } \\
\text { results } \\
\text { (classes } \\
\text { of dips) }\end{array}$ \\
\hline wave 1 & $b-\Phi$ & 0.72 & 0.93 & -117.9 & 38.3 & $b-\Phi$ \\
\hline wave 2 & $c-\Phi$ & 0.65 & 0.79 & -135.2 & 89.7 & $c-\Phi$ \\
\hline wave 3a & $a-\Phi$ & 0.59 & 0.49 & -173.2 & 43.1 & $a-\Phi$ \\
\hline wave 5 & $c a-\Phi$ & 0.77 & 0.47 & -145.5 & 69.5 & $c a-\Phi$ \\
\hline wave 6a & $c-\Phi$ & 0.67 & 0.59 & -135.2 & 89.7 & $c-\Phi$ \\
\hline wave 7 & $a-\Phi$ & 0.59 & 0.49 & -173.5 & 42.9 & $a-\Phi$ \\
\hline wave 8 & $a-\Phi$ & 0.61 & 0.41 & -165.4 & 41.2 & $a-\Phi$ \\
\hline wave 11a & $b-\Phi$ & 0.9 & 0.66 & -129.8 & 40.8 & $b-\Phi$ \\
\hline wave 12 & $3-\Phi$ or & 0.99 & 0.72 & - & - & $\begin{array}{c}\text { - } \Phi-\Phi \text { or } \\
a b-\Phi\end{array}$ \\
\hline wave 13 & $a-\Phi$ & 0.74 & 0.95 & -150.4 & 35.7 & $a-\Phi$ \\
\hline wave 14c & $c-\Phi$ & 0.59 & 0.77 & -134.2 & 89.1 & $c-\Phi$ \\
\hline wave 15 & $c-\Phi$ & 0.70 & 0.89 & -134.2 & 88.8 & $c-\Phi$ \\
\hline
\end{tabular}

Sliding window_Classified window - Phase- $a$--Phase- $b$ - -Phase- $c$

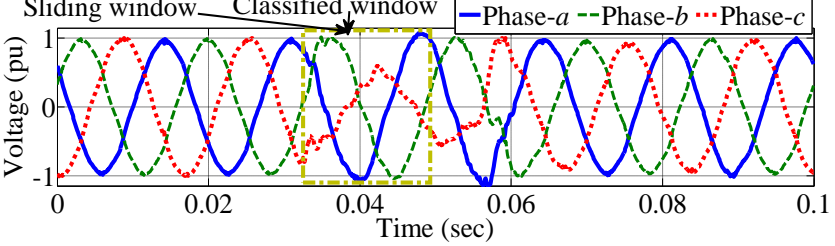

Fig. 10. Recorded waveform wave 15 collected from [15], used for the validation of the proposed approach.

\section{B. Validation of the Proposed Method using Real Measure- ment Data}

Voltage dips, measured in Belgian transmission grid as presented in [16], and voltage swell, recorded from a medium voltage network [11], are used to validate the proposed algorithm. The phasors, illustrated in Table XI, are extracted from the waveform stored during the occurrence of a voltage dip or swell. For classification of each type of dips/swells, the in-phase and out-of-phase projected voltage phasors are obtained by following the procedure presented in Fig. 4; the projected phasors are then passed through the proposed polarization ellipse technique. The whole classification process is conducted in three stages as described in section IIIA. The first stage is disregarded since the PE parameters $\left(A_{y} / A_{x}\right), A_{x}$ and $A_{y}$ do not fall in the groups of balanced dip or no dip condition (see Fig. 5 and Table XII). Therefore, in order to explore the last two stages of classification, the waveform of dip-type $\mathrm{D}$ is taken as an example. Applying the PE parameters, $|\psi|$ and $\varphi$, see Fig. 6, reveals that the "class of dip" is $a-\Phi$. Likewise, applying the PE parameters $-A_{x}$ and $\theta$ in Fig. 7, implies that the type of dip is D. Other two types of dips, $\mathrm{C}$ and $\mathrm{E}$, are classified and characterized using similar approach. Moreover, for H-type swell, applying the PE parameters, $|\psi|$ and $\varphi$, see Fig. 9, reveals that the "group of swell" is $a b-\Phi$ which is denoted as $A B \_$swell_H type. The test results, presented in Table XII, specify the successful classification of $b c \_$dip_E type, $c a \_$dip_C type, $a$ dip_D type and $A B \_$swell_H type.

TABLE XI

PHASORS OF 3-PHASE VOLTAGES RECORDED DURING DIPS/SWELLS

\begin{tabular}{|c|c|c|c|}
\hline Type & $\boldsymbol{V}_{\boldsymbol{a}}$ & $\boldsymbol{V}_{\boldsymbol{b}}$ & $\boldsymbol{V}_{\boldsymbol{c}}$ \\
\hline $\mathrm{C}$ & $0.838<5.2^{\circ}$ & $0.982 \angle-117.9^{\circ}$ & $0.876<115.3^{\circ}$ \\
\hline $\mathrm{D}$ & $0.726<1.7^{\circ}$ & $0.964 \angle-114.6^{\circ}$ & $0.958<116.9^{\circ}$ \\
\hline $\mathrm{E}$ & $0.977<1.2^{\circ}$ & $0.286<-166.6^{\circ}$ & $0.365<124.2^{\circ}$ \\
\hline $\mathrm{H}$ & $1.52 \angle-15.1^{\circ}$ & $1.56<-135^{\circ}$ & $0.08<118.2^{\circ}$ \\
\hline
\end{tabular}

TABLE XII

CLASSIFICATION OF VOLTAGE DIPS/SWELLS WITH RECORDED DATA

\begin{tabular}{|c|c|c|c|c|c|c|}
\hline \multirow{2}{*}{$\begin{array}{l}\text { Dip/Swell } \\
\text { type }\end{array}$} & \multicolumn{5}{|c|}{ Polarization Ellipse parameters } & \multirow{2}{*}{$\begin{array}{c}\text { Classified } \\
\text { dips/swells }\end{array}$} \\
\hline & $A_{1} / A_{x}$ & $A_{x}$ & $\theta^{\circ}$ & $\varphi^{\circ}$ & $|\psi|^{\circ}$ & \\
\hline $\mathrm{C}$ & 0.91 & 1.15 & 126.1 & -139.6 & 55.9 & $c a \operatorname{dip} \mathrm{C}$ \\
\hline $\mathrm{D}$ & 0.85 & 1.17 & 121.2 & -142.9 & 34.7 & $a \_$dip_D \\
\hline$E$ & 0.4 & 0.99 & 117.9 & -101.4 & 77.1 & $b c$ dip_E \\
\hline $\mathrm{H}$ & 0.58 & 1.822 & 175.6 & -135.7 & 89.3 & $A B \_s w e l l \_H$ \\
\hline
\end{tabular}

C. Validation of the Proposed Algorithm using Unbalanced Dips associated with Phase Angle Jump

According to [10], the impedance angle or maximum phase angle jump of $-10^{\circ}$ to $+10^{\circ}$ is found in typical transmission system faults, whereas phase angle jumps of $-40^{\circ}$ to $-60^{\circ}$ occur with faults in distribution lines. In agreement with this, the real cases, which are considered in this sub-section, possess phase angle jump of less than $60^{\circ}$.

In [2] and [10], several test events of voltage dips including single- $\Phi$ and double- $\Phi$ dips are presented. Among those test events, six critical events, which include large phase angle jump and rotation due to loading effects, are used to validate the proposed algorithm. These six events are presented in Table XIII. Event 1 is associated with single- $\Phi$ voltage dip [2]. Event 2 illustrates the scenario when phase " $a$ " experiences a dip of fifty percent with a phase-angle jump of $-30^{\circ}$ in a solidly grounded systems [10]. Event 3 represents a similar case to Event 2 with a larger phase-angle jump, i.e., $-40^{\circ}$ [10]. Event 4 represents the " $b c$ " phase dip incorporating $50 \%$ voltage drop along with phase-angle jump of $-40^{\circ}$. Event 5 and 6 are originated from Event 4 by considering the dynamics of the load associated with voltage drops and rotation [10]. Using the proposed algorithm, it is revealed in column 6 of Table XIII that single- $\Phi$ and double- $\Phi$ dips have been classified correctly. The classified dip-types of all six events are also shown in column 7 of Table XIII. Comparative analysis between space vector method presented in [11] and the proposed PE method are conducted on the basis of classification results of these six critical events. Among these six events, space vector method fails to classify four critical events (Events 3-6) influenced by large phase-angle jump; whereas the proposed PE method has successfully classified all six events. Moreover, the proposed method was tested with adding noise (SNR ranging from 20 $\mathrm{dB}$ to $30 \mathrm{~dB}$ ) and harmonic distortion (THD varied from $1 \%$ to $20 \%$ ); under these conditions, it was found that performance of the proposed method was not affected. 
TABLE XIII

Classification of Unbalanced Voltage Dips associated with Phase-Angle Jump, Voltage Drop and Rotation Due to LoAd EFFECTS

\begin{tabular}{|c|c|c|c|c|c|c|c|}
\hline \multirow{2}{*}{ Events } & \multirow{2}{*}{$V_{a}$} & \multirow{2}{*}{$V_{b}$} & \multirow{2}{*}{$V_{c}$} & \multirow{2}{*}{$\begin{array}{l}\text { "Classes" of dips } \\
\text { (ground-truth) }\end{array}$} & \multicolumn{2}{|c|}{ Classified dips (Proposed PE method) } & \multirow{2}{*}{$\begin{array}{l}\text { Classified "Classes" of } \\
\text { dips using [11] }\end{array}$} \\
\hline & & & & & "Classes" & Dip-type & \\
\hline Event 1 & $0.316<-11^{\circ}$ & $0.842 \angle-103.0^{\circ}$ & $0.848<97.7^{\circ}$ & $a-\Phi$ & $a-\Phi$ & $\mathrm{D}$ & $a-\Phi$ \\
\hline Event 2 & $0.497 \angle-30^{\circ}$ & $1.003<-120^{\circ}$ & $1.003 \angle 120^{\circ}$ & $a-\Phi$ & $a-\Phi$ & B & $a-\Phi$ \\
\hline Event 3 & $0.497 \angle-40^{\circ}$ & $1.003<-120^{\circ}$ & $1.003<120^{\circ}$ & $a-\Phi$ & $a-\Phi$ & B & $a b-\Phi$ \\
\hline Event 4 & $1.00 \angle 0^{\circ}$ & $0.847 \angle-157.1^{\circ}$ & $0.397 \angle 123^{\circ}$ & $b c-\Phi$ & $b c-\Phi$ & $\mathrm{E}$ & $c-\Phi$ \\
\hline Event 5 & $0.85 \angle 0^{\circ}$ & $0.717<-157.0^{\circ}$ & $0.338<124^{\circ}$ & $b c-\Phi$ & $b c-\Phi$ & G & $c-\Phi$ \\
\hline Event 6 & $0.851<-20^{\circ}$ & $0.781<-177.1^{\circ}$ & $0.339<103^{\circ}$ & $b c-\Phi$ & $b c-\Phi$ & G & $c-\Phi$ \\
\hline
\end{tabular}

\section{CONCLUSION}

A new method for classification and characterization of voltage dips and swells is proposed in this paper. Using the proposed approach, a total of seven types (A, B, D, F, E, C, and $\mathrm{G}$ ) of dips, which include 19 possible groups of dips, are classified and characterized. Two types of voltage swells (Hand I-type), which include a total of 6 groups, are also classified using the developed method.

The proposed method is designed based on the Polarization Ellipse (PE) parameters, in 3D co-ordinates, extracted from three phase voltage signals. Six PE parameters are extracted from in-phase and out-of-phase projected voltage phasors on $\mathrm{a}-$, b- and c-axis. Based on the PE parameters, the expressions corresponding to seven types of dips and two types of swells, and their decision boundaries are developed. Using the decision boundaries and PE parameters, three-stage

\section{REFERENCES}

[1] M.A.S. Masoum, S. Jamali, N. Ghaffarzadeh, "Detection and Classification of Power Quality Disturbances using Discrete Wavelet Transform and Wavelet Networks", IET Proceedings on Science, Measurement \& Technology, Vol. 4, Issue 47, pp: 193-205, 2010.

[2] L. Zhang, and M. H. J. Bollen, "Characteristic of voltage dips (sags) in power systems," IEEE Trans. Power Del., vol. 15, no. 2, pp. 827-832, Apr. 2000.

[3] Voltage Characteristics of the Electricity Supplied by Public Distribution Systems, European/British Standard EN (EuroNorms) BS/EN 50160, CLC, BTTF 68-6., Nov. 1994.

[4] IEEE Recommended Practice for Monitoring Electrical Power Quality, IEEE Standard 1159, 1995.

[5] Electricity Supply-Quality Supply, Part 2: Minimum Standards, South African Bureau of Standard. NRS 048-2, 1996, 2002.

[6] Int. Electrotechnical Vocabulary (IEV), IEC Standard 60050, 1999.

[7] S. Z. Djoka, J. V. Milanovic, D. J. Chapman, and M. F. Mcgranaghan, "Shortfalls of existing methods for classification and presentation of voltage reduction events," IEEE Trans. Power Del., vol. 20, no. 2, pt. 2, pp. 1640-1649, Apr. 2005.

[8] M. H. J. Bollen, Understanding Power Quality Problem: Voltage Sag and Interruption. Piscataway, NJ: IEEE, 2000.

[9] M. H. J. Bollen, and L. D. Zhang, "Different methods for classification of three-phase unbalance voltage dips due to faults," Elect. Power Syst. Res., vol. 66, no. 1, pp. 59-69, Jul. 2003.

[10] M. H. J. Bollen, "Algorithms for characterizing measured three-phase unbalanced voltage dips," IEEE Trans. Power Del., vol. 18, no. 3, pp. 937-944, Jul. 2003.

[11] V. Ignatova, P. Granjon, and S. Bacha, "Space vector method for voltage dips and swells analysis," IEEE Trans. Power Del., vol. 24, no. 4, pp. 2054-2061, Oct. 2009. classification algorithm is proposed. The proposed algorithm can effectively classify the balanced dips, six classes (single- $\Phi$ and double- $\Phi$ dips) and their corresponding types of dips $(B$, D, F, E, C and G). Moreover, the developed analytical expressions of decision boundaries require less computation time for cycle by cycle classification. Therefore, the speed of the proposed algorithm is expected to be fast. The proposed algorithm is validated with recorded waveforms extracted from IEEE 1159.2 working group report and real measurement data of Belgian transmission grid. Besides, the proposed PE algorithm is able to classify the unbalanced dips associated with phase angle jump. Thus, the proposed method has a great potential to be used as an important tool for classification and characterization of voltage dips and swells in electricity networks.

[12] T. Carozzi, R. Karlsson, and J. Bergman, "Parameters characterizing electromagnetic wave polarization," Physical Review, vol. 61, pp. 2024$2028,2000$.

[13] P. H. Moon, and D. E. Spencer, Field Theory Handbook: Including Coordinate Systems, Differential Equations, and Their Solutions, Verlag, Springer, 1961.

[14] Yunfu Yang, Ran Tao, and Yue Wang, "A new SINR equation based on the polarization ellipse parameters," IEEE Transactions on Antennas and Propagation, vol.53, no.4, pp.1571-1577, April 2005.

[15] IEEE 1159.2 Working Group, Test waveforms [Available online]. grouper.ieee.org/groups/1159/2/testwave.html

[16] M. H. J. Bollen, P. Goossens, and A. Robert, "Assessment of voltage dips in HV-networks: deduction of complex voltages from the measured rms voltages," IEEE Trans. Power Del., vol. 19, no. 2, pp. 783-790, Apr. 2004

\section{BIOGRAPHIES}

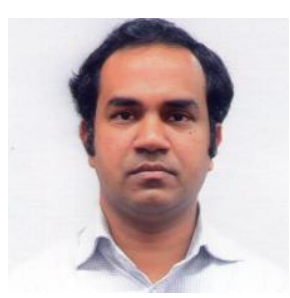

Mollah Rezaul Alam (Std. Member'12) received the B.Sc. degree in Electrical and Electronic Engineering from Bangladesh University of Engineering \& Technology (BUET), Dhaka, Bangladesh in 2005. Currently, he is pursuing the $\mathrm{Ph} . \mathrm{D}$. degree in electrical engineering from the University of Wollongong, New South Wales, Australia.

Prior to starting Ph.D. studies, he was involved in the telecommunication industry in Bangladesh for 5 years, where he worked in the area of Intelligent Network \& Value Added Services of cellular mobile technology. His research interests include computational intelligence, data mining, fault detection, classification and analysis considering the impacts of distributed energy resources. 


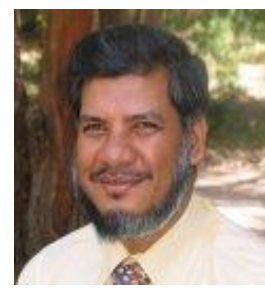

K. M. Muttaqi (M'01, SM'05) received the B.Sc. degree in electrical and electronic engineering from Bangladesh University of Engineering and Technology (BUET), Bangladesh in 1993, the M.Eng.Sc. degree in electrical engineering from University of Malaya, Malaysia in 1996 and the Ph.D. degree in Electrical Engineering from Multimedia University, Malaysia in 2001. Currently, he is an Associate Professor at the School of Electrical, Computer, and Telecommunications Engineering, and member of Australian Power Quality and Reliability (APQRC) at the University of Wollongong, Australia. He was associated with the University of Tasmania, Australia as a Research Fellow/Lecturer/Senior Lecturer from 2002 to 2007, and with the Queensland University of Technology, Australia as a Research Fellow from 2000 to 2002. Previously, he also worked for Multimedia University as a Lecturer for three years. He has more than 18 years of academic experience and has authored or coauthored around 200 papers in international journals and conference proceedings. His research interests include distributed generation, renewable energy, electrical vehicles, smartgrid, power system planning and control. Dr. Muttaqi is an Associate Editor of the IEEE TRANSACTIONS ON INDUSTRY APPLICATIONS.

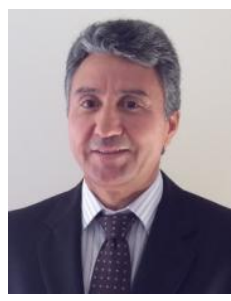

Abdesselam Bouzerdoum (M'89-SM'03) received the M.Sc. and Ph.D. degrees in electrical engineering from the University of Washington, Seattle, USA. In 1991 he joined The University of Adelaide, Adelaide, Australia, and in 1998 he was appointed Associate Professor at Edith Cowan University, Perth, Australia. Since 2004, he has been with the University of Wollongong as Professor of Computer Engineering, where he also served as Head of School of Electrical, Computer \& Telecommunications Engineering (2004-2006) and Associate Dean of Research (2007-2013). From 2009 to 2011, he was a Member of the Australian Research Council College of Experts and served as Deputy Chair of the Engineering, Mathematics and Informatics panel from 2010 to 2011.

Dr. Bouzerdoum was the recipient of numerous awards and prizes, including the Eureka Prize for Outstanding Science in Support of Defence or National Security in 2011, the Chester Sall Award in 2005, and a Distinguished Researcher Award (Chercheur de Haut Niveau) from the French Ministry of Research in 2001. He has published over 300 technical articles and graduated $34 \mathrm{Ph}$.D. and Research Masters students. He served as Associate Editor for 4 International journals, including IEEE TRANS. SYSTEMS, MAN, AND CYBERNETICS (1999-2006). 